\title{
Morphologically Complex Words in Tai Khrang
}

\author{
Unchalee Singnoi Wongwattana
}

Faculty of Humanities, Naresuan University, Thailand

Copyright $\bigcirc 2018$ by authors, all rights reserved. Authors agree that this article remains permanently open access under the terms of the Creative Commons Attribution License 4.0 International License

\begin{abstract}
This article aims to study morphologically complex words in Tai Khrang spoken in the lower northern part of Thailand to examine their syntactic and semantic associations. Data were collected from Ban Nong Moet (Saen to Subdistrict, Khanuworalakburi District, Kamphaeng Phet Province) and Ban Sa Yai Shi (Nong Sano Subdistrict, Sam Ngam District, Phichit Province) as well as from naturally occurring texts such as local narratives and dialogues on Face book. The result shows that morphologically complex words in Tai Khrang are compounded in two strategies such as syntactic and semi-syntactic compounding strategies. As for the syntactic strategy, it is the most salient word formation in the language that is in a complex and sophisticated manner that could reflect the people worldviews and culture. They are performed in three syntactic categories such as synthetic, verb-verb and noun-noun compounds. The synthetic compounding operates like simple clauses, nominalization, and phrases. The verb-verb strategy displays serial verb constructions. And the noun-noun strategy displays different semantic associations graduating from more to less transparent.
\end{abstract}

Keywords Tai Khrang, Syntactic Compounding, Semi-syntactic Compounding, Reduplication, Similarity, Membership, Opposition

\section{Introduction}

Tai Khrang is a Tai ethnic group located in Thailand that still continues to speak its own language, a member of the Tai-Kadai language family which is closely related to Laotian and Standard Thai. The Khrang ethnic group in the northern part of Thailand maintains their traditional lifestyle and practices. They have many names called by the Thai people (both local and government) in different perspectives such as "Lao Khrang/ Khi-Khrang" (people from Lao who use shellac as a dyeing red color), "Lao Phu Khang" (people who came from the Phu Khang area in Lao), "Lao Wiang" (people who came from the Vientiane in Lao), "Tai Khao" (Tai Khao ethnic group in Lao), "Lao
Ka" (people who talk like crows), "Lao Phut" (Buddhist Lao), "Lao Dan" (Lao people who came to Thailand through Dan Sai District, Loei Province) and "Lao Tao Loeng" (Lao people who live in the land of yellow turtles or the lower northern part of Thailand) [1]. This ethnic group is well-known for their textile called "Teen Chok Sarong", a kind of woman long skirt with the red lower part, as shown in the following figure.

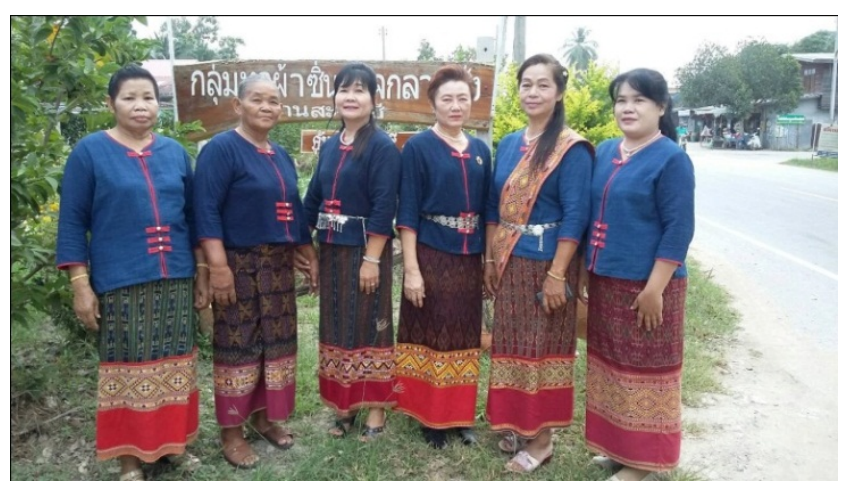

Figure 1. Tai Khrang women and Teen Chok Sarongs

Since the Tai Khrang is a minority group whose traditional practices and beliefs are quite different from those of the majority of people in Thailand, it is also considered an endangered language $\&$ culture and thus needs to be preserved. The best way to preserve these communities is to strengthen their minority rights. Linguistically, most of the previous studies concentrated on phonology and lexical words, e.g., [2], [3], [4], [5], [6] and [1].

The last study reveals that the Tai Khrang dialect in the lower northern part displays 20 consonant phonemes such as $m, n, n, \eta, p, t, k, ?, p^{h}, t^{h}, k^{h}, b, d, f, s, h, t 6, l, w$, and $j$, and 18 vowel phonemes such as $i, i:, e, e:, \varepsilon, \varepsilon ;, \dot{i}, \dot{t}:$, ,, : $a, a:, u, u:, o, o:, \jmath$ and $\jmath:$, including clusters such as $i a$, $\dot{t} a / i a$ and $u a$. There are 5 tones in this area: middle tone (33), high tone (44), rising tone (24/35), falling tone (42) and mid rising-falling tone (343).

However, no study has been elaborately conducted on word formation, a salient linguistic aspect of Tai Khrang, and even of other Tai languages [7], that could well reflect the people mind. We also claim here that most word 
compounds in the Tai languages such as Tai Khrang are relatively transparent in the manner that they are syntactic or could display both grammatical relations and semantic roles/relations in a complex and sophisticated manner, rather than arbitrary grammatical/semantic associations. Within this purpose, the term idiosyncratic or transparent is best accounted for in continuum.

\section{Objectives}

This paper aims to study the combinatorial operations of morphologically complex words in Tai Khrang to examine its linguistic characteristics such as syntactic and semantic aspects.

\section{Methodology and Framework}

Methodologically, the research paper is descriptive. Data were mainly collected from two informants in the lower northern region, one at Ban Nong Moet (Saen To sub-district, Khanuwaralakburi district, Kamphaeng Phet province), and another at Ban Sa Yai Chi (Nong Sano sub-district, Sam Ngam district, Phichit province). Data were also taken from naturally occurring texts such as narratives and daily dialogues found on Face book.

The analysis was mainly conducted on the basis of a functional typological linguistic perspective and cognitive linguistics. Following [8], the analysis makes use of a variety of sources of information aimed at understanding the core formal properties of syntactic constructions such as constituency, hierarchy, grammatical relations and semantic roles. At the same time, the cognitive (conceptual meaning) and communicative (propositional and information discourse coherence or pragmatics) underpinning of grammatical universals are elucidated and underscored, and the interplay between grammar, cognition and communication is outlined. Primary grammatical markers involve coding devices such as morphology and the sequential order of words or morphemes (intonation and rhythmics are also included in spoken communication). Although it (grammar-as-code) is located wholly in the phrase or clause, its functional scope is not primarily about the propositional information couched in the clause in which it resides. Rather, grammar is predominantly about the coherence relations between the propositional (clause) and its wider discourse context.

Particularly, the simple or basic clause in this framework - as the main theoretical implication in this article - is the main, declarative, affirmative, active clause. It is the most frequent clause-type in human discourse and is thus an unmarked syntactic construction. Such a clause-type has to have a privileged cognitive position used in a particular functional domain. It serves as the reference point for grammatical description. Describing the various types of simple clauses is tantamount to describing the various types of verbs, or predications, (states, events, and actions) used in language. Simple clauses are thus defined in terms of their two matching templates or frames, one semantic frame of participant roles, the other syntactic frame of grammatical roles. Clause types other than the basic one are seen as variations. The various clause-types found in the grammar of a language are nothing but differential grammatical packaging of propositional-semantic contents in different discourse-pragmatic functional domains. Compound words are considered as lexical constructions that have imitated a variety of clause types and phrases, either basic ones or variants.

This article also employs perspectives of cognitive linguistics such as "category" and "conceptualization". In [9], categorization is far more complex than those things are categorized together on the basis of what they have in common. For example, the Australian aboriginal language Dyirbal has a category, balan that includes women, fire, and dangerous things as the title of the book. It also includes birds that are not dangerous, as well as animals such as platypus, bandicoot and echidna. Categorization is viewed as the most basic to our thought, perception, action, and speech. Every time we see, reason, perform, or speak/hear something as a kind of thing, we are categorizing. An understanding of how we categorize is central to any understanding of how we think and how we function, and therefore central to an understanding of what makes us human. Within cognitive psychology, [10] has made it an issue, called prototype theory, a framework in the study of categories which have best examples, called "prototypes". For example, robin is a prototype of birds in America; water buffalo is a prototype of working animals in the traditional Thai culture, and so on. The approach to prototype theory suggests that human categorization is essentially a matter of both human experience (of perception, motor activity and culture) and imagination (metaphor, metonymy and mental imagery). [9] associated the prototype effects to the sources called Idealized Cognitive Models (ICM): the knowledge which is organized by means of structures and sources of category structures and prototype effects. An example of ICM is Fillmore's concept of a frame [11]. ICM varies in its complexity: simple, complex and cluster. Later cognitive linguists - e.g., [12] - had questioned the universality of metaphors as early suggested by Lakoff that, he noted that the cultural context is important in interpreting metaphors and that majority of metaphors are culture specific. This has consequently led to the stressing of the importance of culture in conceptual metaphor, while early cognitive linguistics had concerned with structural and orientational metaphors [13]. [14] further discussed the different types of metaphors and posited that there are image metaphors, called 'social-cultural metaphors' because the two domains are associated social-culturally associated. 


\section{Result and Discussion: Combinatorial Operations}

Combinatorial operations that should account for the difference in the contribution of the constituent morphemes in the Tai Khrang language are found as compounding in a number of grammatical characteristics addressed here as syntactic compounding and semi-syntactic compounding. Among these word strategies, syntactic compounding is the most salient type that obviously reflects the people worldviews and culture.

\subsection{Syntactic Compounding}

As already known, the term "compounding" generally refers to the method or strategy where two or more totally different occurring words are picked up to form a compound word which refers to a new thing or idea that is different from any of the original words or the composition of the original words. The new word meaning is idiosyncratic (peculiar to the individual), rather than transparent (straight forward) like a phrase. We, however, would claim here that most word compounds in Tai Khrang are relatively transparent in the manner that they are syntactic or could display grammatical relations and semantic roles/relations, rather than arbitrary grammatical/semantic associations. As stated in [7], the term idiosyncratic or transparent, however, is best accounted for in continuum, rather than two clear-cut conceptual poles. We could still see the track, obviously in the degree of transparency, from which the new meaning is metaphorically created. The following examples display the continuum of compounds in Tai Khrang from more transparent (less idiosyncratic) as in (1a) to more idiosyncratic (less transparent) compound words as in (1c), where an intermediate is in (1b).

1. $k^{h \hat{\imath}}:-h \check{u}:{ }^{1} \quad$ excretion-ear
'earwax'

$$
\begin{aligned}
& \text { b. } k^{h} \hat{\imath}:-h \bar{\varepsilon}: \text { excretion-armpit } \\
& \text { 'underarm odor' } \\
& \text { c. } k^{h} \hat{\imath}:-k a d \bar{t} a n \text { excretion-moon } \\
& \text { 'earthworm' }
\end{aligned}
$$

In (1a), the whole compound word is transparent or similar to a syntactic unit like noun phrases. In (1b), the second member, $/ h \bar{\varepsilon}: /$, still keeps the original meaning, 'armpit', whereas the first member, $/ k^{h} \hat{\imath}$ :/ has lost the original meaning 'excretion/feces'. In (1c), the compound word is highly idiosyncratic: it is difficult to guess its meaning from its members.

Syntactic compounding refers to a word formation strategy used to create a word compound where its members are in a syntactic relation just like clausal (conveying argument structures) or phrasal (conveying the head-modifier relation) constituents are. Similarly to other Tai languages; for instance, Thai [7], Tai Dam [15] and the like, syntactic compound words in Tai Khrang are basically formed in three ways: synthetic, verb-verb and noun-noun, which are different in terms of syntactic and semantic associations.

\subsubsection{Synthetic compounding}

Synthetic compounding is the word building strategy that imitates clauses. Structurally, a word compound of this type employs a clause form or structure, and thus looks pretty much like a clause which conveys the SVO word order regardless of the different levels. Consider the following example where a synthetic compound word in (2a) and a clause in (2b) are compared, with their tree diagrames.

2. a. nák-tī:-mūaj

person-hit-boxing

'boxer'

b. $k h \hat{\jmath} j t \bar{\imath}: m \bar{u} a j$

I hit boxing

'I fight (with the fist).'

1 Tonal transcriptions in this article are presented with diacritical markers: 
Word level

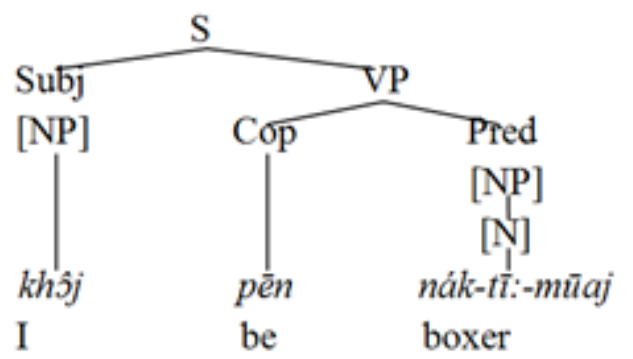

Clause level

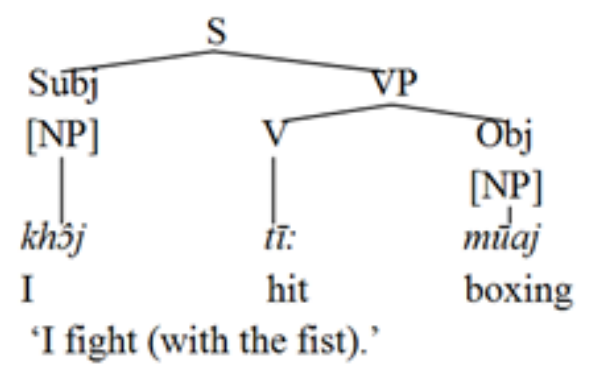

Figure 2. Different levels of clause and compound word

Also, compound members convey argument structures, the systematic mapping of grammatical roles (that is, subject, object, indirect object, predicate and adverbial) and semantic roles (that is, agent, patiant, dative, instrument, locative, and so on) between the main verb and arguments or participants (nominal constituents), just like clausal constituents do. In the example (2), the clause /khôj $t \bar{l}: m \bar{u} a j \mathrm{key} /$ in (2b) displays the argument structure where the subject /khojj/ is an agent, the transitive verb $/ t \bar{i}$ :/ is an action and the object $/ m \bar{u} a j /$ is a patient. Similarly, the compound word /nák-tī:-mūaj/ in (2a) exactly displays such the argument structure: that is, /nák-/plays the agent subject role, /tì:/displays the action and /müaj/ plays the patient object role in the word level.

Synthetic compounds are found in two formal structures: noun head and verb head compounds. Regarding the argument structures of this compounding type, we have found that they reveal various mappings of syntactic and semantic roles (in the noun head form such as subject-agent, subject-dative, subject-instrument, subject-patient, object-patient, object-locative, adverbial-temporal, and so on and in the verb head form such as object-patient, object-locative, predicate-adjective and so on) with the verb members just like those of simple clauses. This thus results in a variety of synthetic compound patterns as exemplified below.

3. a. subject/agent-verb

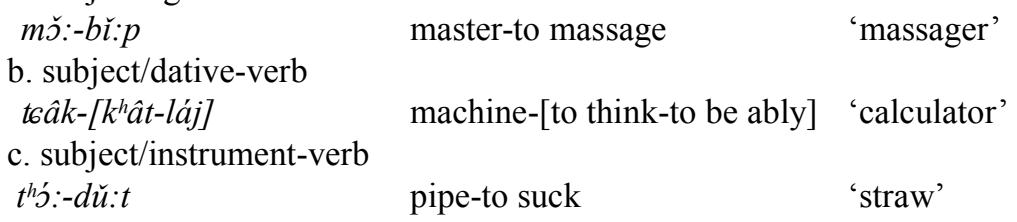

d. subject/patient of state-verb

bāj-jájjż:n leaf-to be sustainable 'warranty'

e. object/patient of change-verb

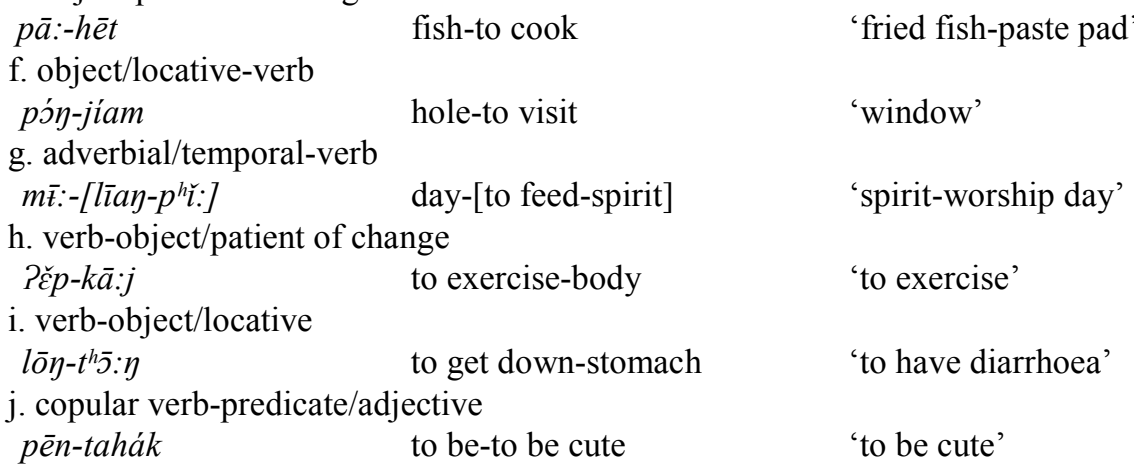

The syntactic and semantic associations of morphologically complex words also are limited in the same manner as those of clauses. That is, the actor can occur as the subject only, the patient as the subject and direct object, the dative as the subject, direct object and indirect object, and the other semantic roles as the indirect objects [8].

Besides the finite-clause forms, it seems that compound words are in forms similar to nominalization. In such compounds, the head functions like a nominalizer and thus could be called a compound nominalizer $(\mathrm{CN})-\mathrm{a}$ grammaticalized unit that forms a compound from a non-nominal category such as clausal or verbal. This is a morpheme such as $/ P \bar{l}: /$, originated from a superordinate noun referring to 'female human', as exemplified below.

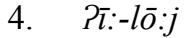
CN-to sprinkle
'watering can' 
In addition, many compound words have imitated or been lexicalized from noun phrases as shown in (5a) and verb phrases in (5b), conveying the noun and verb heads respectively.

5. a. head noun-modifier
$n \bar{a} m-[t a ́: j-s i: k]$
b. auxiliary-head verb
$k^{h} i:-t^{h}{ }^{\swarrow}:$
water-[under-humus] 'waste water'
often-to be niggard 'to be niggard'

\subsubsection{Verb-verb compounding}

Verb-verb or serial verb compounding should be separated from the verb phrase compounding discussed above since it conveys a different set of syntactic and semantic association. Syntactically, while the verb phrase compounding type display the syntactic relation such as modifier-head, the verb-verb type displays the head-head construction where a number of semantic relations are conveyed; namely, activity-objective as in (6a), direction-activity as in (6b) and manner-activity as in (6c) on the one hand and less-prototypical ones such as head-complement as in (6d) and head-preposition as in (6e) on the other hand [16].

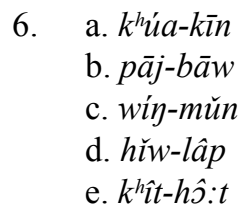

$\begin{array}{ll}\text { to fry without oil-to eat } & \text { 'to fry } \\ \text { to go-to be light } & \text { 'to go pee' } \\ \text { to run-to rotate } & \text { 'turbine' } \\ \text { be hungry for-to sleep } & \text { 'to be sleepy' } \\ \text { to think-to arrive } & \text { 'to miss' }\end{array}$

\subsubsection{Noun-noun compounding}

Noun-noun or nominal compounding is a salient word-creation strategy in Tai Khrang where two nouns or nominal units which are encoded as head-modifier are semantically associated in various patterns. To be better construed, the semantic patterns would be classified in three different formulas in terms of transparency degree levels such as X- $x \rightarrow \mathrm{x}$, $\mathrm{X}-\mathrm{y} \rightarrow \mathrm{X}_{\mathrm{y}}$ and $\mathrm{x}-\mathrm{y} \rightarrow \mathrm{z}$, graduating from more transparent to less transparent. The syntactic and semantic associations of this compounding type thus could be shown as below.

\section{Semantic structure: I. $\mathrm{X}-\mathrm{x} \rightarrow \mathrm{x}$ \\ II. $\mathrm{X}-\mathrm{y} \rightarrow \mathrm{X}_{\mathrm{y}}$ \\ III. $\mathrm{x}-\mathrm{y} \rightarrow \mathrm{z}$}

\section{Syntactic structure : head-modifier}

Figure 3. Frames of noun-noun compounds

\subsubsection{Formula I: $\mathrm{X}-\mathrm{x} \rightarrow \mathrm{x}$}

A number of noun-noun compounds in Tai Khrang display the semantic formula $\mathbf{X}-\mathbf{x} \rightarrow \mathbf{x}$, where the first/head member is the class/higher term ${ }^{2}$ of the second member which could stand for the whole compound itself. The pattern could be separated into four different manners according to the semantic feature of the head parts; namely, generic, shape, gender, whole and excretion heads.

\section{a. Generic Head}

Lakoff states that "Every time we see something as a kind of thing, for example, a tree, we are categorizing. Whenever we reason about kinds of things - chairs, nations, illnesses, any kind of thing at all - we are employing categories"[9]. With evidence in the analysis of 'eating' terms in Thai, [17] concludes that, in order to understand individual things in the world, we have to understand them not only in terms of themselves but also as categories. This implies that the words of a language can be understood via the concept of categorization rather than only in terms of individual words.

Typically, people categorize spatial/concrete entities into animate and inanimate and further categorize animate into human and animal [8], as shown in a simply taxonomic diagram below.

2 According to [18], class terms are different from classifiers. The difference would obviously be predicted on the grounds that class terms occur with their classified nouns in lexicalized compounds, while classifiers occur with their classified terms in nonce syntactic constructions, so that it is perhaps unnecessary to connect this with any significant difference in semantic function. 


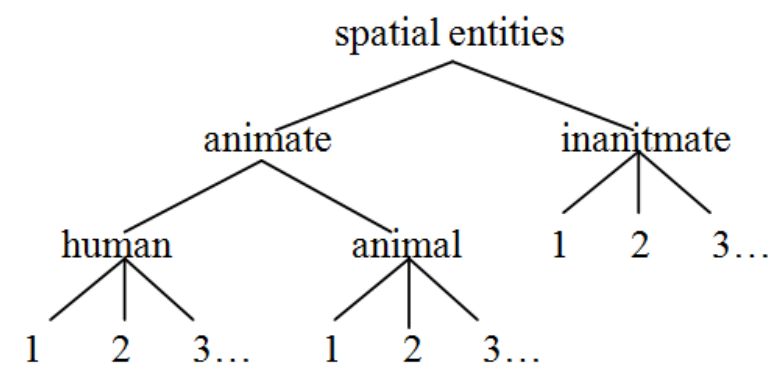

Figure 4. General taxonomic categorization of spatial entities

Similarly to other Tai languages, such as Thai, Tai Dam and the like [7,15], a large number of noun-noun compounds Tai Khrang display a portion of folk taxonomic categorization in the way that the class-term head is a superordinate term followed by a subordinate term as the other member. It is the subordinate member that displays the meaning of the compound since the head is too basic or broad to present a communicative meaning, as illustrated in the following example.

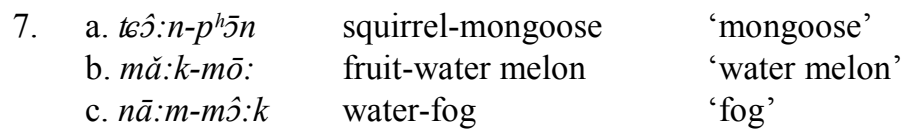

\section{b. Shape head}

Rather than a subordinate term, the class-term head would be the shape class term of the other member, as shown below.
8. a. $t^{h} \bar{i} n-w \bar{u} a$
lump-beef
'beef'
b. kì:p-lép
hoof-nail
'horse/cow's nail'
c. hŭa-kathīam
head shape-garlic 'garlic'

\section{c. Gender head}

A number of class-term head is the gender of the other member which display various kind of identities including, even though inconsistently, kin, animal, fruit, important object and normal object, as respectively shown below.

9. a. kin
PT̄:-ph':
female-father
'father'
b. animal
female-millipede
'millipede'
c. fruit
bâk-thápt $t^{h} \bar{m}$
male-pomegranate
'pomegranate'
d. important object

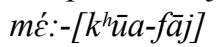
mother-kitchen
'cooking area'
e. normal object
Pī:-dă:p
female-sword
'sword'

\section{d. Whole head}

A number of class-term head display the whole of a part presented by the other member, as exemplified in (10), where the part member conveys a communicative meaning.

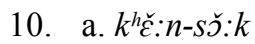
arm-elbow
'elbow'
b. $t \bar{o} n-t \bar{o}$ :
oneself-body
'body'

\section{e. Excretion head}

Last but not least, there are many compounds whose head member is the excretion $/ k^{h} \hat{\imath}: /$ to present the negative interpretation of the other member, as shown below.
11. a. $k^{h \hat{\imath}:-k a b \bar{t}:}$
excretion-navel 'navel'
b. $k^{h} \hat{\imath}:-f i a \eta$
excretion-straw 'straw'
c. $k^{h} \hat{\imath}:-j a ̆: k j a \bar{j} j$
excretion-cobweb 'cobweb' 


\subsubsection{Formula II: $\mathrm{x}-\mathrm{y} \rightarrow \mathrm{x}_{\mathrm{y}}$}

There are a number of compounds in the noun-noun type displaying the semantic formula $\mathbf{x}-\mathbf{y} \rightarrow \mathbf{x}_{\mathbf{y}}$, where the two members are not related in the same manner as compounds in formula I. Rather, the second member modifies the head with a more specific information in various characteristics including portion, whole, compared item, shape, color, amount, material, purpose, possessor, possessed item, body part, location, temporal, profession, expertise, nationality and gender.

\section{a. Portion}

A number of compounds in this formula reveal the metonymic model, a basic characteristic of cognition. Metonymy in the sense of cognitive linguistic theory like [9] is the case that salient part or aspect is commonly used to stand for the things as a whole or for some other aspects or part of it. They display that an object name or concept is employed as a compound member for that of the other member to which it is related, or of which it is a part. Examples are shown below.
12. a. lót-tcâk
b. $\left[p^{h} \hat{a} k-k^{h} \check{o}: m\right]-n a ̆: m$
vehicle-machine
'motorcycle'
c. [bâk-thúa]-[fâk-jā: $: w]$
spinach-thorn
'thorny amaranth'
bean-long pod
'long bean'

\section{b. Whole}

Reversely, the modifying member could display the whole of the partial head, as shown below.
13. a. bía-māj
b. kōy-kīan
sprout-tree
wheel-cart
'tree sprout'
'cart wheel'

\section{c. Compared item}

A large number of compounds in this formula reveal the metaphorical model, another basic characteristics of cognition. [9] proposes that metaphor is conceptual, not merely the use of language. It involves people's world view and thought in a social system. It helps us to understand some things in terms of others that we already understand. The compounds in this type show that the second member applies to an object that implies a resemblance with the head/first member. Examples are shown below.
14. a. sabu:-fün
soap-dust
b. $p \bar{a}:-f a ́$ :
fish-jar lit
'powdered detergent'
c. $k^{h}$ anǒm-[nōm-să:w]
dessert-girl breast
'soft-shelled turtle'
'stuffed dough'

\section{d. Shape}

The modifying member is also found displaying the shape of the head when the compound does not refer to a normal-shape object, as shown below.
15. a. khanǒm-sên
b. jā:-sên
snack-line
medicine-line
'vermicelli/rice noodles'
'cigarette'

\section{e. Color}

The modifying member could display the color of the head, as shown below.
16. a. mót-sôm
ant-orange color 'red ant'

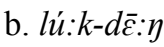
daughter/son-red color
'infant'

\section{f. Amount}

The modifying member could display the collective amount of the item head, as shown below.
17. a. $m \bar{a} j-t^{h} u$ :
b. $p^{h} \hat{a} k-k u m$
stick-pair
vegetable-group
'chopsticks'
'sacred barnar'

\section{g. Material}

The modifying member could display the material of the head, as shown below.
18. thü
bag-rubber
'plastic bag'

\section{h. Purpose}

The modifying member could display the purpose of the head, as shown below. 

19. a. dén-dう̌:k
outdoor-flower
'badminton court'
b. hón-kā:n
room-work
'office'

\section{i. Possessor}

The modifying member could display the possessor of the head, as shown below.

20. a. lép-khit

b. $p \bar{a}:-t c e ́ k$

nail-garuda

fish-Chinese
'Rangoon Creeper'

'a kind of silver barb'

\section{j. Possessed item}

The modifying member could display the possessed item of the head, as shown below.
21. tćáw-tohī:wít
owner-life
'king'

\section{k. Body part}

The modifying member could display the body part which involves the head, as shown below.

22. a. fia $-m \bar{t}$ :

b. tcáw-hǔa

$\begin{array}{ll}\text { loom-hand } & \text { 'loom' } \\ \text { god-head } & \text { 'monk' }\end{array}$

\section{Location}

The modifying member could display the location of the head, as shown below.
23. a. $t^{h} u ́ a-d \bar{\imath} n$
b. láw-pa:
pea-earth
whisky-forest
'ground peanut'
'illegal whisky'

\section{m. Temporal}

The modifying member could display the time of the head, as shown below.
24. $k^{h} \hat{a} w-l \bar{\varepsilon}: \eta$
rice-evening
'dinner/supper'

\section{o. Profession}

The modifying member could display the profession of the human head, as shown below.
25. a. $n \bar{a}: j-k^{h} \bar{u}:$
mister-teacher
'male teacher'
b. $s \bar{a}: w-k^{h} \bar{u}$ :
miss-teacher
'female teacher'

\section{p. Expertise}

The modifying member could display the expertise of the human head, as shown below.

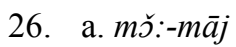
human-wood
'fortune teller'
b. $n \bar{a}: j-p^{h} \bar{a}: s a ̆$ :
mister-language
'interpreter'

\section{q. Nationality}

The modifying member could display the nationality of the head, as shown below.
27. a. $t \bar{a} j-t^{h} \bar{a} j$
b. $k^{h} \bar{a} m-l \bar{a}: w$
people-Thai
'Thai people'
word-Lao
'Laotian language/dialect'

\section{r. Gender}

The modifying member could display the gender of the animate head, as shown below.

28. a. $t \bar{o}:-m \varepsilon \dot{:}$

animal-mother

'female animal'

b. $t \bar{o}:-p^{h}$ :

animal-father

'male animal'

\subsubsection{Formula III: $\mathrm{x}-\mathrm{y} \rightarrow \mathrm{z}$}

There are a relatively small number of compounds displaying the semantic formula $\mathbf{x}-\mathbf{y} \rightarrow \mathbf{z}$, where the two members are not related in the same manners as compounds in formula I and II and the compound meaning is not from the modified head in the same manner as those in formula II. Rather, compound meaning is difficultly related to any compound member. Differently speaking, the compound meaning is highly idiosyncratic or unpredictable from any compound member. Examples are shown below. 

29. a. $k^{h} \bar{a} m-t a w e \bar{n}$
gold-sun
'sunflower'
b. $k^{h} \hat{\imath}:-n o ́$ :
excretion-shoot
'ankle'

The study of syntactic word compounding in Tai Khrang reveals that the people intuitively create new words by employing a variety of syntactic aspects constituting their language. Also, the word formation is performed in a variety of complex and sophisticated manners. The taxonomic figure of syntactic compounds in Tai Khrang could be illustrated below.

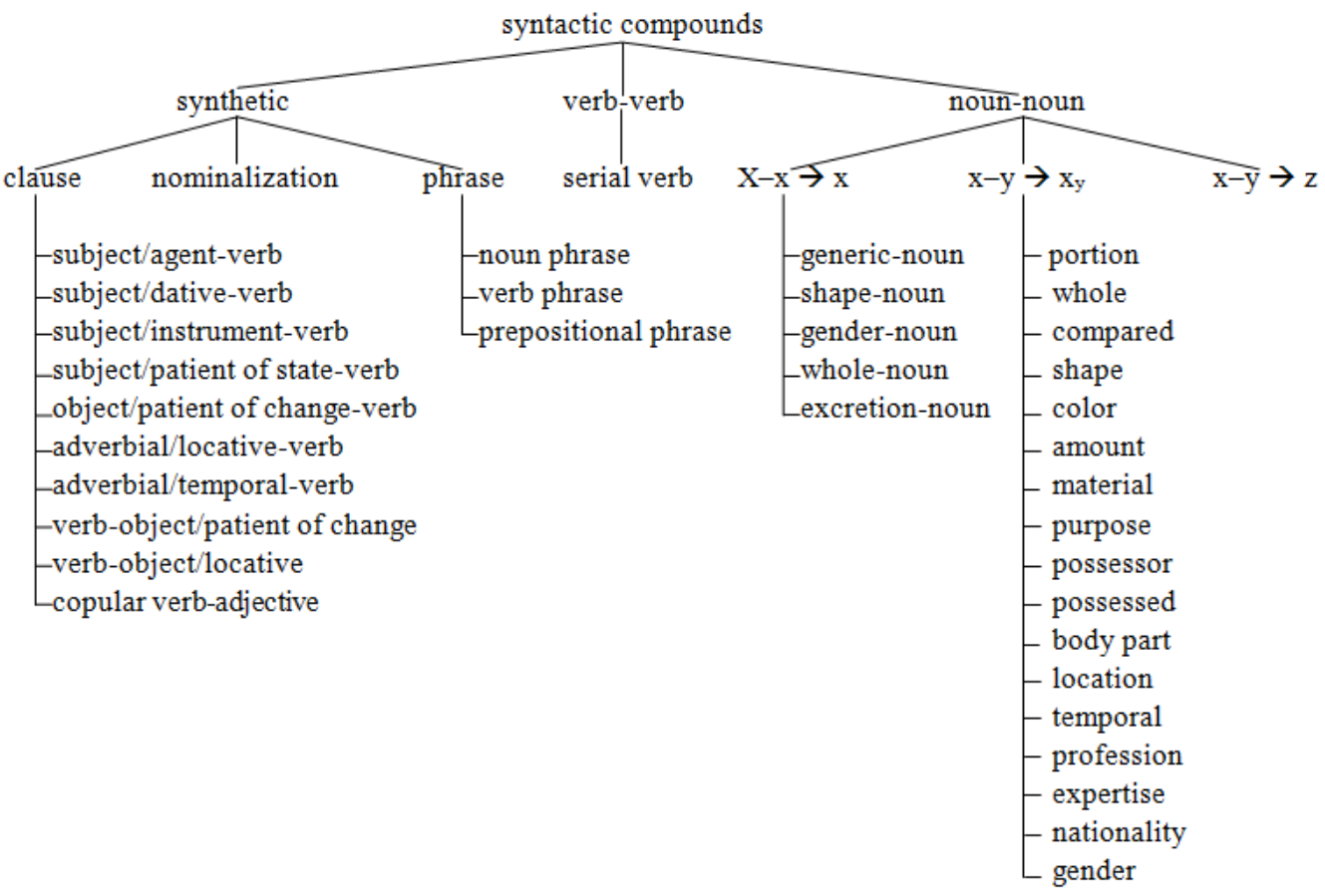

Figure 5. Taxonomic categorization of syntactic compounds in Tai Khrang

\subsection{Semi-syntactic Compounding}

Aside from syntactic compounding strategies where compound members reveal different semantic and grammatical roles in terms of two matching templates or frames, there also are a large number of compounds that show no syntactic relation, leaving semantic aspects displaying the compound templates. That is there is no head-modifier relation operated in compound words - the members are equal in the grammatical status, but display different semantic relations. These word combinations thus called here as semi-syntactic compounds. In the language, semi-syntactic compounds are found in the semantic operations such as reduplication, synonymousness, similarity, membership, and opposition.

\subsubsection{Reduplication}

Semantic reduplication in Tai Khrang could be performed in two different characteristics: without and with morphological change.

\subsubsection{Reduplication without Morphological Change}

Reduplication without morphological change could be further divided into two subtypes according to pholological characteristics such as changing. Redupliction without phonological change is simply formed by repeating the whole word exactly, as shown in (30).

30. $h \bar{\varepsilon}: \eta-h \bar{\varepsilon}: \eta$

strongly-strongly

'somewhat

Reduplication with phonological change have been found as changing of vowel as in (31a), initial consonant as in (31b) and final consonant as in $(31 \mathrm{c})$
31. a. jíam-jā:m
b. sēn-lēn
c. karák-karōn

to visit-to visit

to be stubborn-to be stubborn

to be anxious-to be anxious 'to visit'

'to be stubborn'

'to be anxious' 


\subsubsection{Reduplication with Morphological Change: Synonymousness}

Reduplication with morphological change has been found in a number of compositional characteristics such as similarity, membership, and opposition.

\section{a. Similarity}

Similarity composition is created by combining two words whose meanings are similar, as shown below.
32. a. mú:-phíak
b. tá:j-lá:y
c. $t^{h}:-p^{h} \bar{a}:$

\author{
'gang' \\ 'space under a Tai house' \\ 'pregnant woman'
}

\section{b. Membership}

Membership composition is used to create the superordinate terms of entity sets. It is also common to other Tai languages such as Thai, but different from those of the western languages [7]. In this type, a morphologically complex word is created by combining two different words that could be in the same semantic category to call that category, as shown below.
33. a. $p^{h}:-s o ́: n$
b. $p^{h}$ : $-n \bar{a}$ :
c. lú:k-táw

father-father's mother

father-mother's younger brother/sister'

son/daughter-breast

\author{
'father-in-law' \\ 'stepfather' \\ 'son/daughter'
}

\section{c. Opposition}

Opposition composition is performed by combining two words whose meanings are opposite. Similar to Thai [19], the meaning of the new word can be construed from one compound member or both, as shown below.
34. a. ráp-tó:n
b. $[n \bar{a}-h a ́ k]-[n \bar{a}-s \bar{a} \eta]$
to receive-to round up
to be lovely-to be hateful

\section{Conclusions}

Morphologically complex words in Tai Khrang are composed in many distinctive word characteristics such as syntactic compounding and semi-syntactic compounding such as reduplication, similarity, membership, and opposition. It is the syntactic compounding which is very salient and syntactic. It could be discussed in three basic, structural ways: synthetic, verb-verb and noun-noun compounding. The synthetic strategy is a compound formation that imitates clausal/phrasal constructions such as simple clauses, nominalization, and phrases. Compound words imitating clausal structures conveys various patterns of argument structures such as subject/agent-verb, subject/dative-verb, subject/instrument-verb, subject/patient of state-verb, object/patient of change-verb, adverbial/locative-verb, adverbial/temporal-verb, verb-object/patient of change, verb-object/locative, and copular verb-adjective. The verb-verb strategy displays a different synthetic relation such as serial verb construction. The noun-noun strategy displays three semantic aspects that could be formulated as $\mathrm{X}-\mathrm{x} \rightarrow \mathrm{x}, \mathrm{x}-\mathrm{y} \rightarrow \mathrm{x}_{\mathrm{y}}$ and $\mathrm{x}-\mathrm{y} \rightarrow$ $\mathrm{z}$, graduating from more transparent to less transparent. The first formula contributes to a number of head-difference semantic relations such as generic-noun, shape-noun, gender-noun, whole-noun and excretion-noun. The second formula provides various noun-noun patterns that display different semantic domains inherited in the second member such as portion, whole, compared, shape,

\begin{abstract}
'to welcome'
'to be cute'
\end{abstract}

color, amount, material, purpose, possessor, possessed, body part, location, temporal, profession, expertise, nationality and gender. The last formula reveals less-transparent noun-noun compounds in a relatively small number.

We could see that most word compounds in the Tai languages such as Tai Khrang are relatively transparent in the way that they are syntactic or could display both grammatical relations and semantic roles/relations in a complex and sophisticated manner, rather than arbitrary grammatical/semantic associations. Also, the term idiosyncratic or transparent is best accounted for in continuum.

The complicated strategies seen in Tai Khrang word compositions have implied that the Tai Khrang culture, like every other culture, is prestigious in its value. No culture is more prestigious than others. Realizing this could be the best way for multi-cultural countries, as stated by [20],

"Regarding culture as the concept, every community or country is equally prestigious. No one is more prestigious than others since cultures vary and local cultures are not centralized and are accepted in their own values. This is the best way to carry on multi-culture communities and make them stronger and durable." 


\section{REFERENCES}

[1] Wongwattana, Unchalee S. "Tai Khrang" ethnicity in the lower northern region: Identity and variety, Journal of Community Development Research (Humanities and Social Sciences), Vol.10, No. 2, 2017.

[2] Daecha, Wilailuck. Comparative Study of Phonological Systems of 6 Tai Languages Spoken in Tha Tako District, Nakhonsawan Province, M.A. Thesis, Chulalongkorn University, 1987.

[3] Kamman, Wichit. Lao Khrang Phonological System at Ban Rai District, Uthai Thani Province, Master Thesis, Silapakorn University, 1992.

[4] Watcharaporn, Wanleeya. A Study of Final Particles in Law Khrang, Master Thesis, Mahidol University. 1991.

[5] Nakorn, Chen. Word Syntactic System in Lao Khrang Ban Wanglao, Nongkrot Subdistrict, Meang District, Nakorn Sawan Province, Master Thesis, Silapakorn University, 2001.

[6] Burusphat, Somsonge et al. Ethnic group and language map in the west region of Thailand, Journal of Language and Culture Vol.30 No 2, 84-114, 2011.

[7] Singnoi, Unchalee. Nominal Compound Words: Science and Art in Thai Word Creation, Chulalongkorn University Press, 2005 .

[8] Givón, Talmy. Syntax I and II. John Benjamins Publishing, 2001.

[9] Lakoff, George. Women, Fire, and Dangerous Things: What Categories Reveal about the Mind, The University of Chicago Press, Chicago and London, 1987.
[10] Rosch, Eleanor. Natural Categories. Cognitive Psychology 4, 328-350, 1973.

[11] Fillmore, Charles J. Frame semantics. In Linguistics in the Morning Calm, ed. by The Linguistic Society of Korea, 111-137. Soeul: Hanshin, 1982b.

[12] Kövecses, Z. Metaphor in Culture. Universality and Variation. New York: Cambridge University Press, 2005.

[13] Lakoff, George and Mark Johnson. Metaphor We Live By, University of Chicago Press, Chicago, 1980.

[14] Grady. Theories are buildings revisited, Cognitive Linguistics, 8-4, pp. 267-290, 1997.

[15] Wongwattana, Unchalee S. Word compounding in Tai Dam: A reflection of people world view and culture, Journal of Mekong Societies, Vol.12, Issue 2 May-October, 2016.

[16] Wongwattana, Unchalee S. Verbs in series in Thai: Serial verbs or others?, Journal of Language and Culture, Vol.31, Issue 2, 36-61, 2012.

[17] Singnoi, Unchalee. Eating terms: What the category reveals about the Thai mind, Manusya: Journal of Humanities, Vol.9, No.1, 82-109, 2006.

[18] DeLancey, Scott. Toward a history of Tai classifier systems, In Craig, C. (ed.) Noun Classes and Categorization, John Benjamins Publishing Company, Amsterdam Philadelphia, 437-451, 1986.

[19] Ngamkasem, Poonpong and Unchalee Singnoi. Antonymous doublets in Thai, Journal of Humanities, Vol.9, No.3, 1-16, 2012.

[20] Wasi, Praved. Cultural-wisdom Heritage and the National Security, National Culture Conference "Cultural Research 2" on 12 July 2012, Bangkok.

i The article is a part of the research project "Language Wisdom and Inheritance of Tai Ethnic Groups in the Lower Northern Part: A New Paradigm in the Study of Thai Regional Dialects and Literatures", funded by National Research Council of Thailand (NRCT) in the year 2015-2017. 